\title{
The synthesis and antimycobacterial properties of 4-(substituted benzylsulfanyl)pyridine-2-carboxamides
}

\author{
Věra Klimešová, ${ }^{\text {a* }}$ Petra Herzigová, ${ }^{a}$ Karel Palát, ${ }^{a}$ Miloš Macháček, ${ }^{a}$ Jiřina Stolař́íková, \\ Hans-Martin Dahse, ${ }^{c}$ and Ute Möllmann ${ }^{c}$ \\ ${ }^{a}$ Department of Inorganic and Organic Chemistry, Faculty of Pharmacy, Charles University, \\ Heyrovského 1203, Hradec Králové, 500 05, Czech Republic \\ ${ }^{b}$ Regional Institute of Public Health, Partyzánské nám. 7, Ostrava, 702 00, Czech Republic \\ ${ }^{c}$ Leibniz Institute for Natural Product Research and Infection Biology - Hans Knoell Institute, \\ Beutenbergstr. 11a, D-07745 Jena, Germany \\ E-mail: vera.klimesova@faf.cuni.cz
}

Dedicated to Prof. Rainer Beckert on the occasion of his $60^{\text {th }}$ birthday

\begin{abstract}
4-(Substituted benzylsulfanyl)pyridine-2-carboxamides 6 were synthesized by a three-step synthesis starting from 4-chloropyridine-2-carboxylic acid and substituted benzyl thiols, with the exception of nitroderivatives. The compounds were evaluated for their anti-TB activity against M. tuberculosis, non-tuberculous mycobacteria (M. kansasii and M. avium), and MDR strains of M. tuberculosis. The activities expressed as the minimum inhibitory concentration (MIC) fall into the range of $8-250 \mu \mathrm{mol} / \mathrm{L}$. The substances exhibited similar activities against both sensitive and resistant strains.
\end{abstract}

Keywords: 4-Benzylsulfanyl derivatives, pyridine-2-carboxamides, antimycobacterial activity, cytotoxicity

\section{Introduction}

Tuberculosis (TB), caused by Mycobacterium tuberculosis, continues to be a major threat to global public health, killing more than 1.8 million people in 2009. It has been estimated that in addition nearly 1 billion more people will be infected with TB in the next 20 years. The incidence rate of TB reached a peak worldwide in 2005; however, the total number of new cases continues to slowly rise due to population growth. ${ }^{1}$ The control of TB is seriously threatened due to explosive spread of the HIV epidemic, along with the increasing emergence of multidrug- 
resistant tuberculosis (MDR-TB) and extensively drug-resistant tuberculosis (XDR-TB). The increased incidence of MDR-TB and XDR-TB shows the need for further research. ${ }^{2}$

As a result of our anti-TB project, several papers have been published in which both synthesis and biological activity assessments have been described for a large number of 2- and 4alkylsulfanyl derivatives of substituted pyridines (Figure 1) $3,4,5,6$.
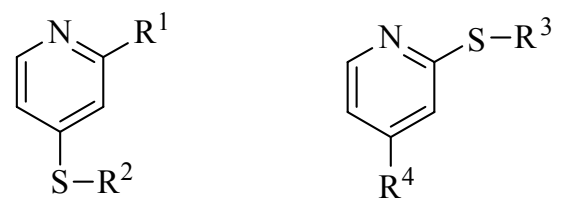

$\mathrm{R}^{1}=\mathrm{CN}, \mathrm{CSNH}_{2}, \mathrm{CONHNH}_{2} ; \mathrm{R}^{2}=$ alkyl, substituted benzyl

$\mathrm{R}^{3}=$ substituted benzyl; $\mathrm{R}^{4}=\mathrm{CN}, \mathrm{CSNH}_{2}$

Figure 1. General structures of model compounds.

For this project we have synthesized a set of 4-(substituted benzylsulfanyl)pyridine-2carboxamides with the goal of finding further conclusions in structure-activity relationships in the group of 4-(benzylsulfanyl)-2-substituted pyridine derivatives. The prepared compounds were evaluated against Mycobacterium tuberculosis, non-tuberculous mycobacteria - M. avium and M. kansasii, as well as against four multi-drug resistant strains of M. tuberculosis.

\section{Results and Discussion}

\section{Chemistry}

The initial pyridine derivative for the synthesis of the desired sulfides $\mathbf{6}$ was selected on the basis of molecular modeling. We computed 3D models of the 4-chloropyridine-2-carboxamide and the 4-chloropyridine-2-carboxylate anion (Figure 2). The higher location of the LUMO orbital on the position 4 on pyridine ring enabled us to predict a carboxylate anion as a suitable reactant for the nucleophilic substitution. Thus, we used 4-chloropyridine-2-carboxylic acid $\mathbf{1}$ in alkaline conditions for the coupling with substituted benzyl thiols 2. 


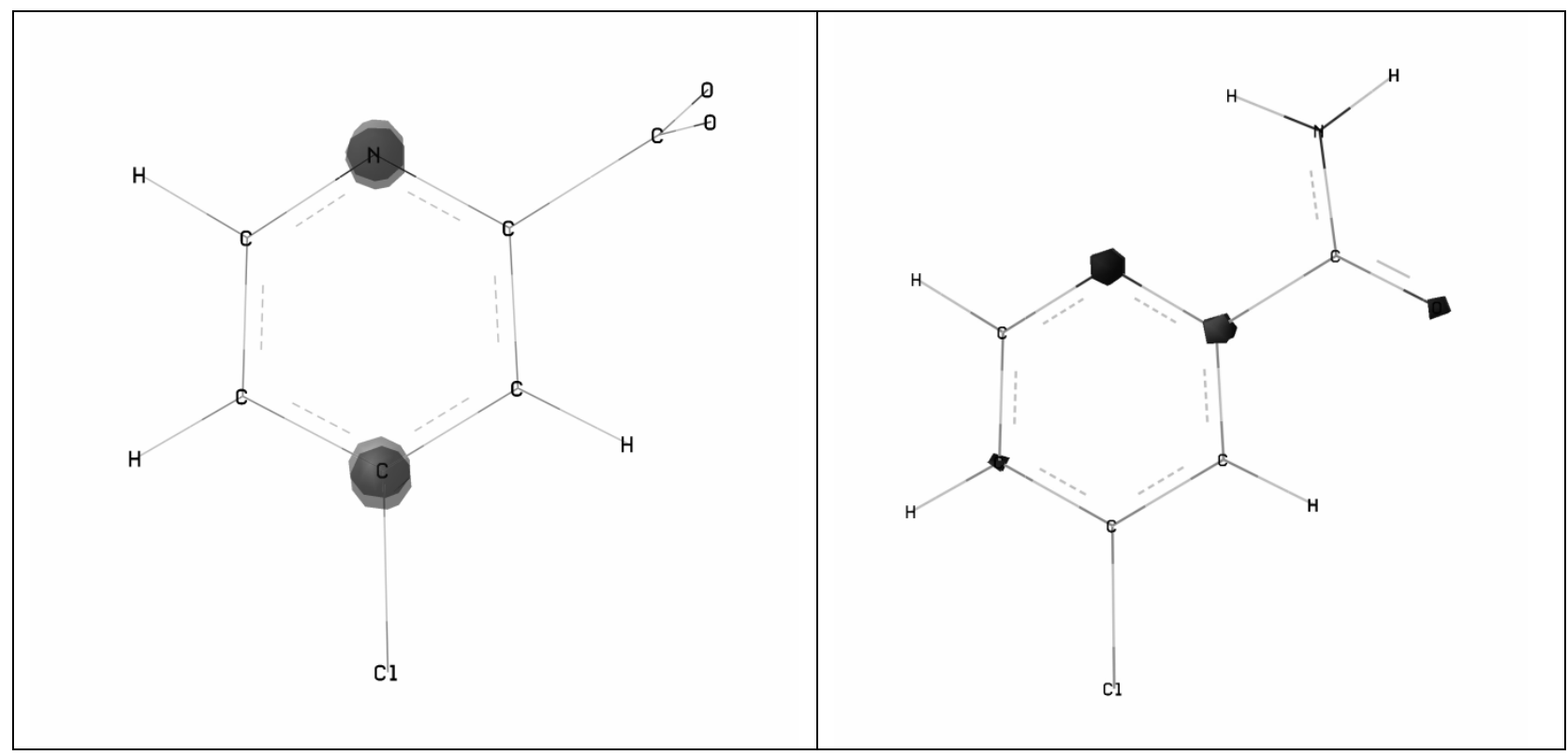

Figure 2. The dislocation of LUMO orbitals in the 4-chloropyridine-2-carboxylate anion and the 4-chloropyridine-2-carboxamide (RB3LYP/6-311+G(d,p) level, software Gaussian03W, v. 6.1; Gaussian, Inc.).

The initial 4-chloropyridine-2-carboxylic acid 1 was prepared according to the literature ${ }^{7}$, the benzyl thiols 2 being prepared by heating appropriate benzyl halides with thiourea in ethanol, and the resulting S-alkylisothiouronium salts hydrolyzed by a sodium hydroxide solution. First, the reaction of $\mathbf{1}$ with unsubstituted benzyl thiol $\mathbf{2 a}$ was studied. Nucleophilic substitution was carried out under the various conditions, the reaction conditions and yields given in Table 1. Namely, reaction in DMF and basic medium $\left(\mathrm{CH}_{3} \mathrm{ONa}\right)$ had not given the appropriate product 3a in good yield after 2 days. Practically the same result occurred when the reaction was carried out in DMF with $\mathrm{K}_{2} \mathrm{CO}_{3}$, using a copper catalyst. Recently, Takahiro and Toshiaki ${ }^{8}$ reported an efficient Pd-catalyzed carbon-sulfur bond formation reaction for aryl thiols with aryl halides using $\mathrm{Pd}_{2}(\mathrm{dba})_{3}$ and a phosphine ligand (Xantphos). Under these conditions, the reaction of 1 with unsubstituted benzyl thiol 2a carried out in dioxane and $\mathrm{i}-\mathrm{Pr}_{2} \mathrm{NEt}$ gave the compound 3a in good yield. In this way the set of desired compounds 3 were prepared by this reaction. The process required 9 to $12 \mathrm{~h}$, depending on the thiol, and furnished products 3 in 32 to $68 \%$ yields (Scheme 1).

Table 1. Reaction conditions and yields of synthesis $\mathbf{3 a}$

\begin{tabular}{ccc}
\hline Reaction conditions & $\begin{array}{c}\text { Temperature / } \\
\text { time }\end{array}$ & Yield (\%) \\
\hline $\mathrm{DMF}, \mathrm{CH}_{3} \mathrm{OH}, \mathrm{Na}$ & r.t. $/ 2$ days & $<5$ \\
$\mathrm{DMF}, \mathrm{K}_{2} \mathrm{CO}_{3}, \mathrm{Cu}$ & $160^{\circ} \mathrm{C} / 6 \mathrm{~h}$ & 15 \\
Dioxane, i-Pr $\mathrm{Pr}_{2} \mathrm{NEt}, \mathrm{Xantphos}, \mathrm{Pd}_{2}(\mathrm{dba})_{3}$ & $110^{\circ} \mathrm{C} / 12 \mathrm{~h}$ & 62 \\
\hline
\end{tabular}


<smiles>O=C(O)c1ccccn1</smiles><smiles>O=C(O)c1cc(Cl)ccn1</smiles>

1

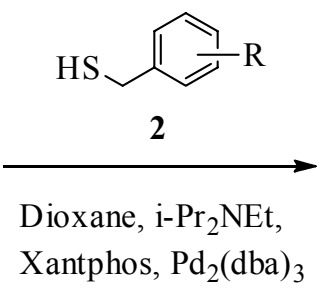

Dioxane, i- $\mathrm{Pr}_{2} \mathrm{NEt}$
Xantphos, $\mathrm{Pd}_{2}(\mathrm{dba})_{3}$<smiles>[R]c1ccc(CSc2ccnc(C(=O)O)c2)cc1</smiles>

3

Scheme 1. Synthesis of 4-(substituted benzylsulfanyl)pyridine-2-carboxylic acids 3a-m.

Table 2. Substituents $\mathrm{R}$ in compounds 2, 3, 5, and $\mathbf{6}$

\begin{tabular}{|c|c|c|c|}
\hline $2,3,5,6$ & $\mathrm{R}$ & $2,3,5,6$ & $\mathrm{R}$ \\
\hline $\mathbf{a}$ & $\mathrm{H}$ & i & $4-\mathrm{CH}_{3}$ \\
\hline b & $3-\mathrm{Cl}$ & $\mathbf{j}$ & $3-\mathrm{CF}_{3}$ \\
\hline c & $4-\mathrm{Cl}$ & $\mathbf{k}$ & $4-\mathrm{CF}_{3}$ \\
\hline d & $3-\mathrm{F}$ & l & $3-\mathrm{CN}$ \\
\hline $\mathbf{e}$ & $4-F$ & $\mathbf{m}$ & $3-\mathrm{OCH}_{3}$ \\
\hline f & $3-\mathrm{Br}$ & $\mathbf{n}$ & $4-\mathrm{NO}_{2}$ \\
\hline g & $4-\mathrm{Br}$ & $\mathbf{0}$ & $3,5-\left(\mathrm{NO}_{2}\right)_{2}$ \\
\hline h & $3-\mathrm{CH}_{3}$ & & \\
\hline
\end{tabular}

This procedure failed for the derivatives with the nitro group on the benzyl moiety. Nitro derivatives 3n and 3o were prepared by hydrolysis in $60 \%$ sulfuric acid of earlier prepared sulfide-nitriles 4 . Sulfides 4 were obtained by reacting 2-cyanopyridine-4-isothiouronium chloride with the nitrobenzyl chlorides in DMF and $\mathrm{CH}_{3} \mathrm{ONa}$ (Scheme 2) ${ }^{4}$.

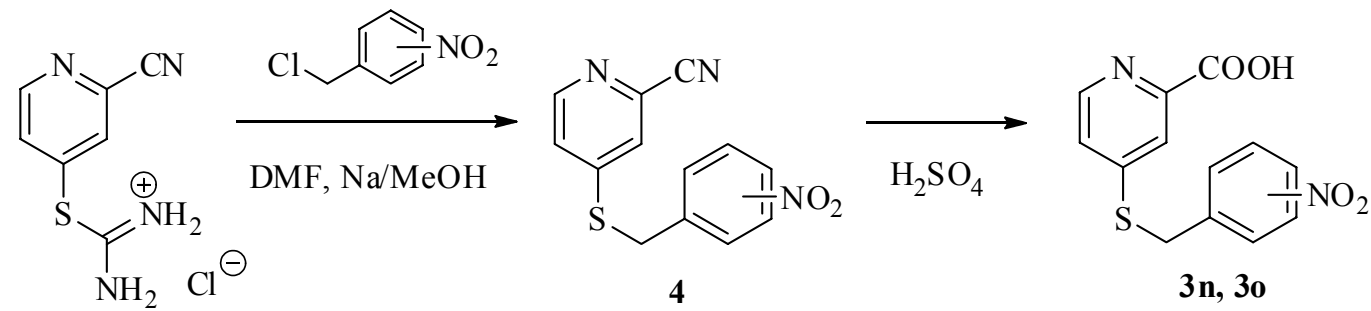

Scheme 2. Synthesis of 4-(nitrobenzylsulfanyl)pyridine-2-carboxylic acids 3n, 30.

The key intermediates 3 were further converted to sulfide-esters 5 by a reaction with $\mathrm{SOCl}_{2}$ in ethanol. The subsequent amonolysis of $\mathbf{5}$ with ammonia gas at ambient temperature for 10-15 $\mathrm{h}$ afforded the desired set of compounds 6 (Scheme 3 ). 


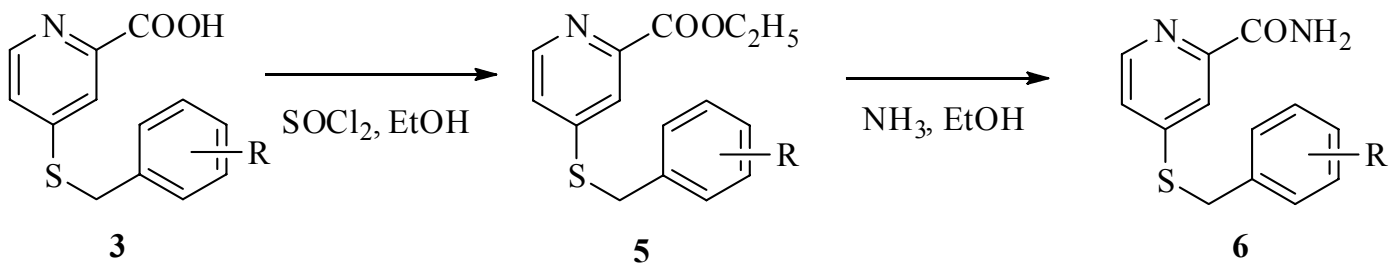

Scheme 3. Synthetic route to the desired amides 6.

\section{Biological activity}

The compounds 6 were screened for their in vitro antimycobacterial activity against $M$. tuberculosis and non-tuberculous mycobacteria - M. kansasii and M. avium. The minimum inhibitory concentrations (MICs) given in $\mu \mathrm{mol} / \mathrm{L}$ are summarized in Table 3. In several cases (denoted >), the MIC could not be determined due to the limited solubility of the compounds in the test medium. As a reference, isoniazid (INH) was included in the assay.

Table 3. In vitro antimycobacterial activity of 4-(benzylsulfanyl)pyridine-2-carboxamides 6 expressed as $\mathrm{MIC}(\mu \mathrm{mol} / \mathrm{L})$

\begin{tabular}{ccccc}
\hline Compound & \multicolumn{4}{c}{ Strains } \\
\hline $\mathbf{6}$ & M. tuberculosis & M. kansasii & M. kansasii & M. avium \\
& My 331/88 & My $235 / 80$ & $6509 / 96$ & My 330/88 \\
& $14 d / 21 d$ & $7 d / 14 d / 21 d$ & $7 d / 14 d / 21 d$ & $14 d / 21 d$ \\
\hline $\mathbf{a}$ & $>125 / 250$ & $62 / 62 / 125$ & $32 / 62 / 125$ & $>62 />62$ \\
$\mathbf{b}$ & $62 / 125$ & $16 / 32 / 62$ & $32 / 62 / 62$ & $62 />62$ \\
$\mathbf{c}$ & $32 / 62$ & $32 / 32 / 32$ & $16 / 32 / 62$ & $32 / 32$ \\
$\mathbf{d}$ & $125 />250$ & $62 / 62 / 62$ & $62 / 125 / 125$ & $>62 />62$ \\
$\mathbf{e}$ & $125 / 125$ & $32 / 62 / 62$ & $32 / 62 / 62$ & $62 / 125$ \\
$\mathbf{f}$ & $32 / 32$ & $16 / 32 / 62$ & $8 / 16 / 32$ & $16 / 32$ \\
$\mathbf{g}$ & $62 / 62$ & $16 / 32 / 62$ & $8 / 8 / 16$ & $32 / 32$ \\
$\mathbf{h}$ & $>62 />62$ & $16 / 32 / 32$ & $32 / 62 />62$ & $62 />62$ \\
$\mathbf{i}$ & $62 />62$ & $16 / 32 / 32$ & $32 / 62 />62$ & $62 />62$ \\
$\mathbf{j}$ & $32 / 62$ & $8 / 16 / 32$ & $32 / 32 / 32$ & $62 / 62$ \\
$\mathbf{k}$ & $16 / 32$ & $16 / 16 / 32$ & $16 / 16 / 32$ & $32 / 32$ \\
$\mathbf{I}$ & $125 / 125$ & $32 />62 />125$ & $125 / 250 / 500$ & $>125 />250$ \\
$\mathbf{m}$ & $62 / 62$ & $16 / 62 / 62$ & $32 />62 />125$ & $>62 />62$ \\
$\mathbf{n}$ & $>62 />62$ & $>32 />62 />125$ & $>16 />32 />62$ & $>125 />125$ \\
$\mathbf{0}$ & $32 / 32$ & $8 / 32 / 32$ & $8 / 8 / 16$ & $32 / 62$ \\
INH & $0.5 / 1$ & $>250 />250 />250$ & $2 / 2 / 4$ & $>250 />250$ \\
\hline
\end{tabular}

Generally, all tested compounds showed moderate in vitro activity against all tested mycobacterial strains. The MIC values vary predominantly between 16 and $62 \mu \mathrm{mol} / \mathrm{L}$. By 
comparing their MIC values with those of INH, the compounds were less active against $M$. tuberculosis My 331/88 and M. kansasii 6509/96, however more active against M. kansasii My 235/80 and $M$. avium My 330/80. In contrast to INH, the compounds were similarly active against both $M$. kansasii strains. The advantage of the prepared compounds is their activity against $M$. avium. This strain exhibits an extreme resistance to all current antimycobacterial drugs. The obtained MIC values of compounds $\mathbf{6}$ indicate that substitution in the benzyl moiety with various electron-accepting or electron-donating substituents does not affect the antimycobacterial activity. The same conclusion was obtained for the previously synthesized 4-(substituted benzylsulfanyl)pyridine-2-carbohydrazide. ${ }^{6}$ But, the antimycobacterial activity is strongly connected with the substituents on the pyridine moiety. In our previous paper we described the antimycobacterial activity of 4-(substituted benzylsulfanyl)pyridine-2carbohydrazides ${ }^{6},-2$-carbonitriles ${ }^{4}$, and -2-carbothioamides ${ }^{4}$. The newly prepared derivatives 6 have comparable activity with carbonitrile derivatives, but did not reach the activity of carbohydrazides and carbothioamides. Carbohydrazides are two to four times more active against M. tuberculosis My 331/88 and M. kansasii My 235/80 (MICs range within 4-32 $\mu \mathrm{mol} / \mathrm{L}$ ) than compounds 6 and comparable active against clinical isolate M. kansasii 6509/96 and M. avium My 330/80 (MICs range within 16-62 $\mu \mathrm{mol} / \mathrm{L}$ ). Carbothioamides (MICs ranging within 4-16 $\mu \mathrm{mol} / \mathrm{L}$ ) appear to be the most active derivatives against all tested strains.

The selected compounds $\mathbf{6}$ were further evaluated against four multidrug-resistant strains of M. tuberculosis isolated from TB patients. These results are summarized in Table 4.

Table 4. In vitro antituberculotic activity against MDR M. tuberculosis (MIC expressed in $\mu \mathrm{mol} / \mathrm{L})$

\begin{tabular}{ccccc}
\hline Compound & \multicolumn{4}{c}{ Strains } \\
\hline $\mathbf{6}$ & M. tuberculosis & M. tuberculosis & M. tuberculosis & M. tuberculosis \\
& $7357 / 1998^{a}$ & $9449 / 2007^{b}$ & $234 / 2005^{c}$ & $8666 / 2010^{d}$ \\
& $14 d / 21 d$ & $14 d / 21 d$ & $14 d / 21 d$ & $14 d / 21 d$ \\
\hline $\mathbf{b}$ & $62 />125$ & $32 />125$ & $125 />125$ & $>125 />125$ \\
$\mathbf{d}$ & $>125 />125$ & $>62 />125$ & $>125 />125$ & $>125 />125$ \\
$\mathbf{f}$ & $>32 />125$ & $>32 />125$ & $>32 />125$ & $>125 />125$ \\
$\mathbf{h}$ & $62 />125$ & $>62 />125$ & $>125 />125$ & $>125 />125$ \\
$\mathbf{j}$ & $62 / 125$ & $62 / 125$ & $62 / 125$ & $>125 />125$ \\
$\mathbf{k}$ & $16 / 62$ & $32 / 62$ & $32 / 62$ & $>62 />62$ \\
$\mathbf{e}$ & $>125 />125$ & $>125 />125$ & $>125 />125$ & $>250 />250$ \\
$\mathbf{0}$ & $32 / 62$ & $>32 />62$ & $32 / 62$ & $62 />62$ \\
\hline
\end{tabular}

${ }^{a}$ Resistant to isoniazid, rifampicin, streptomycin, ethambutol, ofloxacin, and ansamycin. ${ }^{b}$ Resistant to isoniazid, rifampicin, streptomycin, and ansamycin. ${ }^{c}$ Resistant to isoniazid, rifampicin, streptomycin, ethambutol, and ansamycin. ${ }^{\mathrm{d}}$ Resistant to isoniazid, rifampicin, streptomycin, ethambutol, ofloxacin, ansamycin, and clofazimine. 
The evaluated compounds exhibit antimycobacterial activity on both drug-susceptible and drugresistant $M$. tuberculosis strains. The activities are nearly the same against all tested strains. The comparable susceptibility of multi-drug resistant and sensitive strains of $M$. tuberculosis indicates that there is no cross resistance with current antituberculous drugs, thus the target of prepared compounds might be new.

Several compounds were assayed against cell lines HUVEC and K-562 for their antiproliferative effects $\left(\mathrm{GI}_{50}\right.$ : concentration which inhibited cell proliferation by $50 \%$ compared to control), and against HeLa cells for their cytotoxic effects $\left(\mathrm{CC}_{50}\right.$ : a cytotoxic concentration which contains a specific destructive action by $50 \%$ compared to the control; used particularly in referring to the lysis of cells). The cells were incubated with 10 concentrations of the tested compounds. The compounds $\mathbf{6}$ have low to moderate antiproliferative and cytotoxic activity (Table 5).

Table 5. Antiproliferative $\left(\mathrm{GI}_{50}\right)$ and cytotoxic $\left(\mathrm{CC}_{50}\right)$ effect (expressed in $\mu \mathrm{g} / \mathrm{mL}$ )

\begin{tabular}{cccc}
\hline Compound & Huvec $\left(\mathrm{GI}_{50}\right)$ & $\mathrm{K}-562\left(\mathrm{GI}_{50}\right)$ & $\mathrm{HeLa}\left(\mathrm{CC}_{50}\right)$ \\
\hline $\mathbf{6 a}$ & $>50$ & $>50$ & 36.8 \\
$\mathbf{6 c}$ & 41.9 & 20.6 & 49.7 \\
$\mathbf{6 g}$ & 33.1 & 28 & 44.2 \\
$\mathbf{6 i}$ & $>50$ & $>50$ & $>50$ \\
$\mathbf{6 k}$ & 26.5 & 18.9 & 74.9 \\
\hline
\end{tabular}

\section{QSAR study}

For the purpose of this study the MIC values of compounds 6 in $\mu \mathrm{mol} / \mathrm{L}$ (Table 3) were expressed as $\log (1 / \mathrm{MIC})=-\log \mathrm{MIC}$. When the MIC value is marked with $>$, i.e. when the MIC could not be determined exactly, double the MIC value was used. The parameters taken into consideration ( $\log P$, Hammet constant $\sigma$, and energy of HOMO, LUMO orbitals) are summarized in Table 6.

Table 6. Physicochemial and quantum-chemical parameter values of compounds 6

\begin{tabular}{ccccc}
\hline Compound & $\sigma^{9}$ & HOMO & LUMO & $\Delta \log P$ \\
\hline $\mathbf{6 a}$ & 0 & -6.5531 & -1.74154 & 0 \\
$\mathbf{6 b}$ & 0.37 & -6.69786 & -1.84114 & 0.589 \\
$\mathbf{6 c}$ & 0.22 & -6.66575 & -1.84522 & 0.583 \\
$\mathbf{6 d}$ & 0.34 & -6.68997 & -1.82916 & -0.027 \\
$\mathbf{6 e}$ & 0.06 & -6.64671 & -1.83379 & 0.092 \\
$\mathbf{6 f}$ & 0.37 & -6.70194 & -1.84495 & 0.739 \\
$\mathbf{6 g}$ & 0.22 & -6.66603 & -1.84168 & 0.867 \\
$\mathbf{6 h}$ & -0.06 & -6.50657 & -1.7176 & 0.551 \\
$\mathbf{6 i}$ & -0.14 & -6.46929 & -1.7195 & 0.527
\end{tabular}


Table 6. (Continued)

\begin{tabular}{ccccc}
\hline $\mathbf{6 j}$ & 0.46 & -6.79093 & -1.90644 & 0.788 \\
$\mathbf{6 k}$ & 0.53 & -6.8127 & -1.91624 & 0.855 \\
$\mathbf{6}$ & 0.62 & -6.86794 & -1.9859 & -0.576 \\
$\mathbf{6 m}$ & 0.10 & -6.32071 & -1.69555 & 0.067 \\
$\mathbf{6 n}$ & 0.81 & -6.9444 & -2.94049 & -0.224 \\
$\mathbf{6 o}$ & 1.42 & -7.19257 & -3.51465 & -0.384 \\
\hline
\end{tabular}

First, we sought relationships between MIC values and lipophilicity. In the whole set of carboxamides 6, lipophilic parameter $\Delta \log P$ showed poor correlations with the antimycobacterial activities. The analysis through Jackknife residuals of the dependencies indicated the MIC values for two compounds, $\mathbf{6 n}\left(4-\mathrm{NO}_{2}\right)$ and $\mathbf{6 o}\left(3,5-\mathrm{diNO}_{2}\right)$ as outliers. After the outliers have been removed, statistically significant relationships were obtained. As the relationships for different incubation times are similar, only those after 14 days of incubation are presented.

$$
\begin{gathered}
-\log \text { MIC (M. tuberculosis })=0.54( \pm 0.16) \Delta \log P-2.03( \pm 0.092) \\
\mathrm{n}=13, \mathrm{~s}=0.243, \mathrm{r}=0.713 \\
-\log \text { MIC }(\text { M. kansasii })=0.540( \pm 0.060) \Delta \log P-1.80( \pm 0.035) \\
\mathrm{n}=13, \mathrm{~s}=0.092, \mathrm{r}=0.938 \\
-\log \text { MIC (M. kansasii } 6509 / 96)=0.82( \pm 0.14) \Delta \log P-2.00( \pm 0.080) \\
\mathrm{n}=13, \mathrm{~s}=0.213, \mathrm{r}=0.870 \\
-\log \text { MIC }(\text { M. avium })=0.64( \pm 0.11) \Delta \log P-2.04( \pm 0.063) \\
\mathrm{n}=13, \mathrm{~s}=0.168, \mathrm{r}=0.866
\end{gathered}
$$

Antimycobacterial activities of carboxamides $\mathbf{6}$ with the exception of nitro derivatives $\mathbf{6 n}$ and 60 increased with their lipophilicity. The addition of another descriptor, e.g. Hammett constants or quantum chemical parameters (HOMO, LUMO energies), did not significantly improve the statistics of the relationships.

\section{Conclusions}

4-(Substituted benzylsulfanyl)pyridine-2-carboxamides $\mathbf{6}$ were synthesized by a three-step synthesis starting from 4-chloropyridine-2-carboxylic acid and substituted benzyl thiols using Pd-catalyzed cross-coupling reaction carried out in dioxane, i- $\operatorname{Pr}_{2} \mathrm{NEt}$, and Xantphos. The key intermediate sulfide-carboxylic acids $\mathbf{3}$ were converted to sulfide-esters $\mathbf{5}$ by a reaction with $\mathrm{SOCl}_{2}$ in ethanol. The subsequent amonolysis of $\mathbf{5}$ with ammonia gas at ambient temperature afforded the desired compounds 6. Nitroderivatives were prepared from 2-cyanopyridine-4isothiouronium chloride and the nitrobenzyl chlorides in $\mathrm{DMF}$ and $\mathrm{CH}_{3} \mathrm{ONa}$. The prepared 
sulfide-nitriles 4 afforded the key intermediates 3 by hydrolysis in $60 \%$ sulfuric acid. The compounds 6 were evaluated for their anti-TB activity against $M$. tuberculosis, non-tuberculous mycobacteria (M. kansasii and M. avium), and MDR strains of M. tuberculosis. The activities expressed as a minimum inhibitory concentrations fall into the range of $8-250 \mu \mathrm{mol} / \mathrm{L}$. The substances exhibited similar moderate activities against both sensitive and resistant strains of $M$. tuberculosis.

\section{Experimental Section}

\section{Chemistry}

General. The melting points were determined on a Kofler block and are uncorrected. Analytical samples were dried over $\mathrm{P}_{4} \mathrm{O}_{10}$ at $78{ }^{\circ} \mathrm{C}$ or $25{ }^{\circ} \mathrm{C}$ and $2.4-2.6 \mathrm{kPa}$ for 2 hours. Elemental analyses were performed on CHNS-O CE instrument (FISONS EA 1110). IR spectra were obtained on a Nicolet Impact 400 spectrometer in $\mathrm{KBr}$ pellets. NMR spectra were recorded on a Varian Mercury-Vx BB 300 spectrometer operating at $300 \mathrm{MHz}$ for ${ }^{1} \mathrm{H}$ and $75 \mathrm{MHz}$ for ${ }^{13} \mathrm{C}$ with DMSO- $\mathrm{d}_{6}$ as a solvent and TMS as internal standard.

Compounds $\mathbf{3}$ and $\mathbf{5}$ were obtained and characterized in our previous studies ${ }^{6}$; compounds $\mathbf{4}$ are described in our paper ${ }^{4}$.

\section{General procedures for preparation of amides 6}

A two-neck, round-bottom flask equipped with a drying tube and a feeding tube was charged with a corresponding ester $5(1.3 \mathrm{mmol})$ and 3-4 $\mathrm{ml}$ absolute ethanol. Dry ammonia gas was passed into the reaction solution at ambient temperature for 10-15 h. The reaction was monitored by TLC (Merck TLC plates silica gel 60 F254, aluminum back) in chloroform - methanol triethylamine 9:1:1.25. The plates were visualized using UV light. Subsequently, the mixture was left overnight in the refrigerator. The precipitated solid was filtered off and washed with water. The crude product was purified by crystallization with ethanol.

4-(Benzylsulfanyl)pyridine-2-carboxamide (6a). White crystals, yield 42\%, mp. 108-110 ${ }^{\circ} \mathrm{C}$; IR $\left(v_{\max }, \mathrm{cm}^{-1}\right): 3382(\mathrm{~N}-\mathrm{H}), 3276(\mathrm{~N}-\mathrm{H}), 3200(\mathrm{~N}-\mathrm{H}), 1679(\mathrm{C}=\mathrm{O}), 1604\left(\mathrm{NH}_{2}\right) .{ }^{1} \mathrm{H}-\mathrm{NMR}(300$ MHz, DMSO- $\left.d_{6}\right): \delta(\mathrm{ppm}) 8.41\left(1 \mathrm{H}, \mathrm{dd}, J=5.3 \mathrm{~Hz}, J=0.7 \mathrm{~Hz}, \mathrm{H}_{6}\right), 8.10(\mathrm{~s}, 1 \mathrm{H}, \mathrm{NH}), 7.90(1 \mathrm{H}$, $\left.\mathrm{dd}, J=2.0 \mathrm{~Hz}, J=0.7 \mathrm{~Hz}, \mathrm{H}_{3}\right), 7.68(1 \mathrm{H}, \mathrm{s}, \mathrm{NH}), 7.50\left(1 \mathrm{H}, \mathrm{dd}, J=5.3 \mathrm{~Hz}, J=2.0 \mathrm{~Hz}, \mathrm{H}_{5}\right), 7.48-$ $7.43\left(2 \mathrm{H}, \mathrm{m}, \mathrm{H}_{\mathrm{Ar}}\right), 7.38-7.24\left(3 \mathrm{H}, \mathrm{m}, \mathrm{H}_{\mathrm{Ar}}\right), 4.44\left(2 \mathrm{H}, \mathrm{s}, \mathrm{CH}_{2}\right) .{ }^{13} \mathrm{C}-\mathrm{NMR}\left(75 \mathrm{MHz}, \mathrm{DMSO}-d_{6}\right)$ : $\delta(\mathrm{ppm}) 165.8,150.5,150.5,150.2,148.3,136.2,129.1,128.8,127.6,122.9,118.6$, 34.2; Anal. Calcd for $\mathrm{C}_{13} \mathrm{H}_{12} \mathrm{~N}_{2} \mathrm{OS}(244.31) \mathrm{C}, 63.91 ; \mathrm{H}, 4.95 ; \mathrm{N}, 11.47 \%$. Found: C, 63.68; H, 4.82; N, $11.62 \%$.

4-(3-Chlorobenzylsulfanyl)pyridine-2-carboxamide (6b). White crystals, yield 58\%, mp. 131$132{ }^{\circ} \mathrm{C}$; IR $\left(v_{\max }, \mathrm{cm}^{-1}\right): 3389(\mathrm{~N}-\mathrm{H}), 3165(\mathrm{~N}-\mathrm{H}), 1663(\mathrm{C}=\mathrm{O}), 1625\left(\mathrm{NH}_{2}\right) ;{ }^{1} \mathrm{H}-\mathrm{NMR}(300$ MHz, DMSO- $\left.d_{6}\right) \delta(\mathrm{ppm}) 8.42\left(1 \mathrm{H}, \mathrm{dd}, J=5.3 \mathrm{~Hz}, J=0.6 \mathrm{~Hz}, \mathrm{H}_{6}\right), 8.09(1 \mathrm{H}, \mathrm{s}, \mathrm{NH}), 7.89(1 \mathrm{H}$, 
$\left.\mathrm{dd}, J=2.0 \mathrm{~Hz}, J=0.6 \mathrm{~Hz}, \mathrm{H}_{3}\right), 7.69(1 \mathrm{H}, \mathrm{s}, \mathrm{NH}), 7.54\left(1 \mathrm{H}, \mathrm{s}, \mathrm{H}_{\mathrm{Ar}}\right), 7.50(1 \mathrm{H}, \mathrm{dd}, J=5.3 \mathrm{~Hz}, J=$ $\left.2.0 \mathrm{~Hz}, \mathrm{H}_{5}\right), 7.44-7.31\left(3 \mathrm{H}, \mathrm{m}, \mathrm{H}_{\mathrm{Ar}}\right), 4.46\left(2 \mathrm{H}, \mathrm{s}, \mathrm{CH}_{2}\right) ;{ }^{13} \mathrm{C}-\mathrm{NMR}\left(75 \mathrm{MHz}, \mathrm{DMSO}-d_{6}\right) \delta(\mathrm{ppm})$ 165.8, 150.3, 150.0, 148.3, 139.0, 133.3, 130.6, 128.9, 127.7, 127.6, 123.0, 118.7, 33.5; Anal. Calcd for $\mathrm{C}_{13} \mathrm{H}_{11} \mathrm{ClN}_{2} \mathrm{OS}(278.76) \mathrm{C}, 56.01 ; \mathrm{H}, 3.98 ; \mathrm{N}, 10.05 \%$. Found: C, 56.24; H, 4.12; N, $10.23 \%$.

4-(4-Chlorobenzylsulfanyl)pyridine-2-carboxamide (6c). Off-white crystals, yield 40\%, mp. 123-124 ${ }^{\circ} \mathrm{C}$; IR $\left(v_{\max }, \mathrm{cm}^{-1}\right): 3383(\mathrm{~N}-\mathrm{H}), 3279(\mathrm{~N}-\mathrm{H}), 3169(\mathrm{~N}-\mathrm{H}), 1682(\mathrm{C}=\mathrm{O}), 1599\left(\mathrm{NH}_{2}\right)$; ${ }^{1} \mathrm{H}-\mathrm{NMR}\left(300 \mathrm{MHz}, \mathrm{DMSO}-d_{6}\right) \delta(\mathrm{ppm}) 8.41\left(1 \mathrm{H}, \mathrm{dd}, J=5.3 \mathrm{~Hz}, J=0.6 \mathrm{~Hz}, \mathrm{H}_{6}\right), 8.09(1 \mathrm{H}, \mathrm{s}$, $\mathrm{NH}), 7.88\left(1 \mathrm{H}, \mathrm{dd}, J=2.0 \mathrm{~Hz}, J=0.6 \mathrm{~Hz}, \mathrm{H}_{3}\right), 7.68(1 \mathrm{H}, \mathrm{s}, \mathrm{NH}), 7.50-7.45\left(3 \mathrm{H}, \mathrm{m}, \mathrm{H}_{5}, \mathrm{H}_{\mathrm{Ar}}\right)$, 7.42-7.37 (2H, m, $\left.\mathrm{H}_{\mathrm{Ar}}\right), 4.45\left(2 \mathrm{H}, \mathrm{s}, \mathrm{CH}_{2}\right) ;{ }^{13} \mathrm{C}-\mathrm{NMR}\left(75 \mathrm{MHz}, \mathrm{DMSO}-d_{6}\right) \delta$ (ppm) 165.8, 150.3, 150.1, 148.3, 135.5, 132.2, 130.9, 128.8, 123.0, 118.7, 33.4; Anal. Calcd for $\mathrm{C}_{13} \mathrm{H}_{11} \mathrm{ClN}_{2} \mathrm{OS}$ (278.76) C, 56.01; H, 3.98; N, 10.05\%. Found: C, 56.30; H, 3.76; N, 10.28\%.

4-(3-Fluorobenzylsulfanyl)pyridine-2-carboxamide (6d). Off-white crystals, yield 72\%, mp. 144-146 ${ }^{\circ} \mathrm{C}$; IR $\left(v_{\max }, \mathrm{cm}^{-1}\right) 3388(\mathrm{~N}-\mathrm{H}), 3278(\mathrm{~N}-\mathrm{H}), 3160(\mathrm{~N}-\mathrm{H}), 1682(\mathrm{C}=\mathrm{O}), 1619\left(\mathrm{NH}_{2}\right) ;{ }^{1} \mathrm{H}-$ NMR (300 MHz, DMSO-d $) \delta(\mathrm{ppm}) 8.41\left(1 \mathrm{H}, \mathrm{dd}, J=5.3 \mathrm{~Hz}, J=0.6 \mathrm{~Hz}, \mathrm{H}_{6}\right), 8.09(1 \mathrm{H}, \mathrm{s}$, $\mathrm{NH}), 7.89\left(1 \mathrm{H}, \mathrm{dd}, J=2.0 \mathrm{~Hz}, J=0.6 \mathrm{~Hz}, \mathrm{H}_{3}\right), 7.68(1 \mathrm{H}, \mathrm{s}, \mathrm{NH}), 7.50(1 \mathrm{H}, \mathrm{dd}, J=5.3 \mathrm{~Hz}, J=$ $\left.2.0 \mathrm{~Hz}, \mathrm{H}_{5}\right), 7.42-7.28\left(3 \mathrm{H}, \mathrm{m}, \mathrm{H}_{\mathrm{Ar}}\right), 7.14-7.06\left(1 \mathrm{H}, \mathrm{m}, \mathrm{H}_{\mathrm{Ar}}\right), 4.47\left(\mathrm{~s}, 2 \mathrm{H}, \mathrm{CH}_{2}\right) ;{ }^{13} \mathrm{C}-\mathrm{NMR}(75$ MHz, DMSO- $\left.d_{6}\right) \delta(\mathrm{ppm}) 165.8,162.3(\mathrm{~d}, J=244.3 \mathrm{~Hz}), 150.3,150.1,148.3,139.3(\mathrm{~d}, J=7.6$ $\mathrm{Hz}), 130.7$ (d, $J=8.5 \mathrm{~Hz}), 125.2(\mathrm{~d}, J=2.9 \mathrm{~Hz}), 123.0,118.7,115.8(\mathrm{~d}, J=21.9 \mathrm{~Hz}), 114.5(\mathrm{~d}, J$ $=20.9 \mathrm{~Hz}$ ), 33.6; Anal. Calcd for $\mathrm{C}_{13} \mathrm{H}_{11} \mathrm{FN}_{2} \mathrm{OS}$ (262.30) C, 59.53; H, 4.23; N, 10.68\%. Found: C, 59.38; H, 4.05; N, 10.44\%.

4-(4-Fluorobenzylsulfanyl)pyridine-2-carboxamide (6e). Off-white crystals, yield 59\%, mp. 131-135 ${ }^{\circ} \mathrm{C}$; IR $\left(v_{\max }, \mathrm{cm}^{-1}\right) 3378(\mathrm{~N}-\mathrm{H}), 3286(\mathrm{~N}-\mathrm{H}), 3170(\mathrm{~N}-\mathrm{H}), 1687(\mathrm{C}=\mathrm{O}), 1604\left(\mathrm{NH}_{2}\right) ;{ }^{1} \mathrm{H}-$ NMR (300 MHz, DMSO-d $)_{6} \delta(\mathrm{ppm}) 8.41\left(1 \mathrm{H}, \mathrm{dd}, J=5.3 \mathrm{~Hz}, J=0.6 \mathrm{~Hz}, \mathrm{H}_{6}\right), 8.09(1 \mathrm{H}, \mathrm{s}$, $\mathrm{NH}), 7.88\left(1 \mathrm{H}, \mathrm{dd}, J=2.0 \mathrm{~Hz}, J=0.6 \mathrm{~Hz}, \mathrm{H}_{3}\right), 7.68(1 \mathrm{H}, \mathrm{s}, \mathrm{NH}), 7.52-7.47\left(3 \mathrm{H}, \mathrm{m}, \mathrm{H}_{5}, \mathrm{H}_{\mathrm{Ar}}\right)$, 7.21-7.13 (m, 2H, $\mathrm{H}_{\mathrm{Ar}}$ ), $4.44\left(2 \mathrm{H}, \mathrm{s}, \mathrm{CH}_{2}\right) ;{ }^{13} \mathrm{C}-\mathrm{NMR}\left(75 \mathrm{MHz}, \mathrm{DMSO}-d_{6}\right) \delta(\mathrm{ppm}) 165.8,161.6$ $(\mathrm{d}, J=243.9 \mathrm{~Hz}), 150.2,150.3,148.3,132.5(\mathrm{~d}, J=3.0 \mathrm{~Hz}), 131.1(\mathrm{~d}, J=8.2 \mathrm{~Hz}), 123.0,118.6$, $115.6(\mathrm{~d}, J=21.6 \mathrm{~Hz}), 33.4$; Anal. Calcd for $\mathrm{C}_{13} \mathrm{H}_{11} \mathrm{FN}_{2} \mathrm{OS}(262.30) \mathrm{C}, 59.53 ; \mathrm{H}, 4.23$; N, 10.68\%. Found: C, 59.31; H, 4.34; N, 10.88\%.

4-(3-Bromobenzylsulfanyl)pyridine-2-carboxamide (6f). White crystals, yield 75\%, mp. 123$124{ }^{\circ} \mathrm{C}$; IR ( $\left.v_{\max }, \mathrm{cm}^{-1}\right) 3392(\mathrm{~N}-\mathrm{H}), 3285(\mathrm{~N}-\mathrm{H}), 3139(\mathrm{~N}-\mathrm{H}), 1683(\mathrm{C}=\mathrm{O}), 1617\left(\mathrm{NH}_{2}\right) ;{ }^{1} \mathrm{H}-$ NMR (300 MHz, DMSO-d $)_{6} \delta(\mathrm{ppm}) 8.42\left(1 \mathrm{H}, \mathrm{dd}, J=5.3 \mathrm{~Hz}, J=0.6 \mathrm{~Hz}, \mathrm{H}_{6}\right), 8.10(1 \mathrm{H}, \mathrm{s}$, $\mathrm{NH}), 7.89\left(1 \mathrm{H}, \mathrm{dd}, J=2.0 \mathrm{~Hz}, J=0.6 \mathrm{~Hz}, \mathrm{H}_{3}\right), 7.68-7.67\left(2 \mathrm{H}, \mathrm{m}, \mathrm{NH}, \mathrm{H}_{\mathrm{Ar}}\right), 7.51-7.45(3 \mathrm{H}, \mathrm{m}$, $\left.\mathrm{H}_{5}, \mathrm{H}_{\mathrm{Ar}}\right), 7.32-7.27\left(1 \mathrm{H}, \mathrm{m}, \mathrm{H}_{\mathrm{Ar}}\right), 4.46\left(2 \mathrm{H}, \mathrm{s}, \mathrm{CH}_{2}\right) ;{ }^{13} \mathrm{C}-\mathrm{NMR}\left(75 \mathrm{MHz}, \mathrm{DMSO}-d_{6}\right) \delta(\mathrm{ppm})$ 165.8, 150.3, 150.0, 148.3, 139.3, 131.8, 130.9, 130.5, 128.1, 123.0, 122.0, 118.7, 33.4; Anal. Calcd for $\mathrm{C}_{13} \mathrm{H}_{11} \mathrm{BrN}_{2} \mathrm{OS}$ (323.21) C, 48.31; H, 3.43; N, 8.67\%. Found: C, 48.58; H, 3.65; N, $8.84 \%$.

4-(4-Bromobenzylsulfanyl)pyridine-2-carboxamide (6g). White crystals, yield 35\%, mp. 141$143{ }^{\circ} \mathrm{C}$; IR $\left(v_{\max }, \mathrm{cm}^{-1}\right) 3369(\mathrm{~N}-\mathrm{H}), 3151(\mathrm{~N}-\mathrm{H}), 1686(\mathrm{C}=\mathrm{O}), 1603\left(\mathrm{NH}_{2}\right) ;{ }^{1} \mathrm{H}-\mathrm{NMR}(300 \mathrm{MHz}$, DMSO- $\left.d_{6}\right) \delta(\mathrm{ppm}) 8.40\left(1 \mathrm{H}, \mathrm{dd}, J=5.3 \mathrm{~Hz}, J=0.3 \mathrm{~Hz}, \mathrm{H}_{6}\right), 8.08(1 \mathrm{H}, \mathrm{s}, \mathrm{NH}), 7.87(1 \mathrm{H}, \mathrm{dd}, J=$ 
$\left.2.0 \mathrm{~Hz}, J=0.3 \mathrm{~Hz}, \mathrm{H}_{3}\right), 7.67(1 \mathrm{H}, \mathrm{s}, \mathrm{NH}), 7.53-7.50\left(2 \mathrm{H}, \mathrm{m}, \mathrm{H}_{\mathrm{Ar}}\right), 7.47(1 \mathrm{H}, \mathrm{dd}, J=5.3 \mathrm{~Hz}, J=$ $\left.2.0 \mathrm{~Hz}, \mathrm{H}_{5}\right), 7.42-7.39\left(2 \mathrm{H}, \mathrm{m}, \mathrm{H}_{\mathrm{Ar}}\right), 4.42\left(2 \mathrm{H}, \mathrm{s}, \mathrm{CH}_{2}\right) ;{ }^{13} \mathrm{C}-\mathrm{NMR}\left(75 \mathrm{MHz}, \mathrm{DMSO}-d_{6}\right) \delta(\mathrm{ppm})$ $165.8,150.3,150.1,148.3,135.9,131.7,131.2,123.0,120.8,118.7$, 33.5; Anal. Calcd for $\mathrm{C}_{13} \mathrm{H}_{11} \mathrm{BrN}_{2} \mathrm{OS}$ (323.21) C, 48.31; H, 3.43; N, 8.67\%. Found: C, 48.52; H, 3.32; N, 8.44\%.

4-(3-Methylbenzylsulfanyl)pyridine-2-carboxamide (6h). Off-white crystals, yield 59\%, mp. 131-132 ${ }^{\circ} \mathrm{C}$; IR $\left(v_{\max }, \mathrm{cm}^{-1}\right) 3380(\mathrm{~N}-\mathrm{H}), 3280(\mathrm{~N}-\mathrm{H}), 3158(\mathrm{~N}-\mathrm{H}), 1687(\mathrm{C}=\mathrm{O}), 1604\left(\mathrm{NH}_{2}\right) ;{ }^{1} \mathrm{H}-$ NMR $\left(300 \mathrm{MHz}, \mathrm{DMSO}-d_{6}\right) \delta(\mathrm{ppm}) 8.41\left(1 \mathrm{H}, \mathrm{dd}, J=5.3 \mathrm{~Hz}, J=0.6 \mathrm{~Hz}, \mathrm{H}_{6}\right), 8.09(1 \mathrm{H}, \mathrm{s}$, $\mathrm{NH}), 7.90\left(1 \mathrm{H}, \mathrm{dd}, J=2.0 \mathrm{~Hz}, J=0.6 \mathrm{~Hz}, \mathrm{H}_{3}\right), 7.68(1 \mathrm{H}, \mathrm{s}, \mathrm{NH}), 7.48(1 \mathrm{H}, \mathrm{dd}, J=5.3 \mathrm{~Hz}, J=$ $\left.2.0 \mathrm{~Hz}, \mathrm{H}_{5}\right), 7.27-7.19\left(3 \mathrm{H}, \mathrm{m}, \mathrm{H}_{\mathrm{Ar}}\right), 7.10-7.06\left(1 \mathrm{H}, \mathrm{m}, \mathrm{H}_{\mathrm{Ar}}\right), 4.38\left(2 \mathrm{H}, \mathrm{s}, \mathrm{CH}_{2}\right), 2.28(3 \mathrm{H}, \mathrm{s}$, $\left.\mathrm{CH}_{3}\right) ;{ }^{13} \mathrm{C}-\mathrm{NMR}\left(75 \mathrm{MHz}, \mathrm{DMSO}-d_{6}\right) \delta$ (ppm) 165.8, 150.6, 150.2, 148.3, 138.0, 136.0, 129.7, 128.7, 128.3, 126.2, 122.8, 118.5, 34.3, 21.1; Anal. Calcd for $\mathrm{C}_{14} \mathrm{H}_{14} \mathrm{~N}_{2} \mathrm{OS}$ (258.34) C, 65.09; $\mathrm{H}$, 5.46; N, 10.84\%. Found: C, 65.30; H, 5.36; N, 10.57\%.

4-(4-Methylbenzylsulfanyl)pyridine-2-carboxamide (6i). Yellowish crystals, yield 40\%, mp. 116-118 ${ }^{\circ} \mathrm{C}$; IR $\left(v_{\max }, \mathrm{cm}^{-1}\right) 3384(\mathrm{~N}-\mathrm{H}), 3285(\mathrm{~N}-\mathrm{H}), 3193(\mathrm{~N}-\mathrm{H}), 1683(\mathrm{C}=\mathrm{O}), 1601\left(\mathrm{NH}_{2}\right) ;{ }^{1} \mathrm{H}-$ NMR (300 MHz, DMSO-d $)) \delta(\mathrm{ppm}) 8.40\left(1 \mathrm{H}, \mathrm{dd}, J=5.3 \mathrm{~Hz}, J=0.5 \mathrm{~Hz}, \mathrm{H}_{6}\right), 8.09(1 \mathrm{H}, \mathrm{s}$, $\mathrm{NH}), 7.88\left(1 \mathrm{H}, \mathrm{dd}, J=2.0 \mathrm{~Hz}, J=0.5 \mathrm{~Hz}, \mathrm{H}_{3}\right), 7.68(1 \mathrm{H}, \mathrm{s}, \mathrm{NH}), 7.48(1 \mathrm{H}, \mathrm{dd}, J=5.3 \mathrm{~Hz}, J=$ $\left.2.0 \mathrm{~Hz}, \mathrm{H}_{5}\right), 7.38-7.28\left(2 \mathrm{H}, \mathrm{m}, \mathrm{H}_{\mathrm{Ar}}\right), 7.18-7.10\left(2 \mathrm{H}, \mathrm{m}, \mathrm{H}_{\mathrm{Ar}}\right), 4.38\left(2 \mathrm{H}, \mathrm{s}, \mathrm{CH}_{2}\right), 2.26(3 \mathrm{H}, \mathrm{s}$, $\left.\mathrm{CH}_{3}\right) ;{ }^{13} \mathrm{C}-\mathrm{NMR}\left(75 \mathrm{MHz}, \mathrm{DMSO}-d_{6}\right) \delta(\mathrm{ppm}) 165.8,150.6,150.2,148.3,136.9,133.0,129.4$, 129.0, 122.9, 118.6, 34.0, 20.9; Anal. Calcd for $\mathrm{C}_{14} \mathrm{H}_{14} \mathrm{~N}_{2} \mathrm{OS}$ (258.34) C, 65.09; H, 5.46; N, 10.84\%. Found: C, 65.26; H, 5.39; N, 10.98\%.

4-(3-Trifluoromethylbenzylsulfanyl)pyridine-2-carboxamide (6j). Yellowish crystals, yield 58\%, mp. 107-108 ${ }^{\circ} \mathrm{C}$; IR $\left(v_{\max }, \mathrm{cm}^{-1}\right) 3328(\mathrm{~N}-\mathrm{H}), 3191(\mathrm{~N}-\mathrm{H}), 1684(\mathrm{C}=\mathrm{O}), 1616\left(\mathrm{NH}_{2}\right), 1331$ $\left(\mathrm{CF}_{3}\right) ;{ }^{1} \mathrm{H}-\mathrm{NMR}\left(300 \mathrm{MHz}, \mathrm{DMSO}-d_{6}\right) \delta(\mathrm{ppm}) 8.42\left(1 \mathrm{H}, \mathrm{dd}, J=5.3 \mathrm{~Hz}, J=0.6 \mathrm{~Hz}, \mathrm{H}_{6}\right), 8.09$ $(1 \mathrm{H}, \mathrm{s}, \mathrm{NH}), 7.91\left(1 \mathrm{H}, \mathrm{dd}, J=2.0 \mathrm{~Hz}, J=0.6 \mathrm{~Hz}, \mathrm{H}_{3}\right), 7.86-7.82\left(1 \mathrm{H}, \mathrm{m}, \mathrm{H}_{\mathrm{Ar}}\right), 7.80-7.74(2 \mathrm{H}, \mathrm{m}$, $\left.\mathrm{H}_{\mathrm{Ar}}\right), 7.68(1 \mathrm{H}, \mathrm{s}, \mathrm{NH}), 7.68-7.54\left(2 \mathrm{H}, \mathrm{m}, \mathrm{H}_{\mathrm{Ar}}\right), 7.51\left(1 \mathrm{H}, \mathrm{dd}, J=5.3 \mathrm{~Hz}, J=2.0 \mathrm{~Hz}, \mathrm{H}_{5}\right), 4.57$ $\left(2 \mathrm{H}, \mathrm{s}, \mathrm{CH}_{2}\right)$; ${ }^{13} \mathrm{C}-\mathrm{NMR}\left(75 \mathrm{MHz}, \mathrm{DMSO}-d_{6}\right) \delta(\mathrm{ppm}) 165.7,150.3,149.8,148.3,138.1,133.2$, 129.9, 129.4 (q, $J=31.8 \mathrm{~Hz}), 125.7$ (q, $J=3.9 \mathrm{~Hz}), 124.4$ (q, $J=3.9 \mathrm{~Hz}), 124.3$ (q, $J=272.9$ $\mathrm{Hz})$, 123.1, 118.8, 33.5; Anal. Calcd for $\mathrm{C}_{14} \mathrm{H}_{11} \mathrm{~F}_{3} \mathrm{~N}_{2} \mathrm{OS}$ (312.31) C, 53.84; H, 3.55; N, 8.97\%. Found: C, 53.58; H, 3.76; N, 9.11\%.

4-(4-Trifluoromethylbenzylsulfanyl)pyridin-2-carboxamide (6k). White crystals, yield 55\%, mp. 123-124 ${ }^{\circ} \mathrm{C}$; IR $\left(v_{\max }, \mathrm{cm}^{-1}\right) 3378(\mathrm{~N}-\mathrm{H}), 3290(\mathrm{~N}-\mathrm{H}), 3180(\mathrm{~N}-\mathrm{H}), 1682(\mathrm{C}=\mathrm{O}), 1619\left(\mathrm{NH}_{2}\right)$, $1324\left(\mathrm{CF}_{3}\right) ;{ }^{1} \mathrm{H}-\mathrm{NMR}\left(300 \mathrm{MHz}, \mathrm{DMSO}-d_{6}\right) \delta(\mathrm{ppm}) 8.41\left(1 \mathrm{H}, \mathrm{dd}, J=5.3 \mathrm{~Hz}, J=0.6 \mathrm{~Hz}, \mathrm{H}_{6}\right)$, $8.09(1 \mathrm{H}, \mathrm{s}, \mathrm{NH}), 7.90\left(1 \mathrm{H}, \mathrm{dd}, J=2.0 \mathrm{~Hz}, J=0.6 \mathrm{~Hz}, \mathrm{H}_{3}\right), 7.73-7.67\left(5 \mathrm{H}, \mathrm{m}, \mathrm{H}_{\mathrm{Ar}}, \mathrm{NH}\right), 7.51$ $\left(1 \mathrm{H}, \mathrm{dd}, J=5.3 \mathrm{~Hz}, J=2.0 \mathrm{~Hz}, \mathrm{H}_{5}\right), 4.56\left(2 \mathrm{H}, \mathrm{s}, \mathrm{CH}_{2}\right) ;{ }^{13} \mathrm{C}-\mathrm{NMR}\left(75 \mathrm{MHz}, \mathrm{DMSO}-d_{6}\right) \delta(\mathrm{ppm})$ 165.7, 150.3, 149.8, 148.4, 141.5, 129.8, 128.3 (q, $J=31.8 \mathrm{~Hz}), 125.7$ (q, $J=3.7 \mathrm{~Hz}), 124.4$ (q, $J$ $=272.9 \mathrm{~Hz}), 123.0,118.7,33.6$; Anal. Calcd for $\mathrm{C}_{14} \mathrm{H}_{11} \mathrm{~F}_{3} \mathrm{~N}_{2} \mathrm{OS}(312.31) \mathrm{C}$, 53.84; H, 3.55; N, 8.97\%. Found: C, 53.91; H, 3.39; N, 8.78\%.

4-(3-Cyanobenzylsulfanyl)pyridine-2-carboxamide (61). White crystals, yield 60\%, mp. 196$198^{\circ} \mathrm{C}$; IR $\left(v_{\max }, \mathrm{cm}^{-1}\right) 3377(\mathrm{~N}-\mathrm{H}), 3282(\mathrm{~N}-\mathrm{H}), 3161(\mathrm{~N}-\mathrm{H}), 2229(\mathrm{CN}), 1683(\mathrm{C}=\mathrm{O}), 1605$ $\left(\mathrm{NH}_{2}\right) ;{ }^{1} \mathrm{H}-\mathrm{NMR}\left(300 \mathrm{MHz}, \mathrm{DMSO}-d_{6}\right) \delta(\mathrm{ppm}) 8.42\left(1 \mathrm{H}, \mathrm{dd}, J=5.3 \mathrm{~Hz}, J=0.6 \mathrm{~Hz}, \mathrm{H}_{6}\right), 8.09$ 
$(1 \mathrm{H}, \mathrm{s}, \mathrm{NH}), 7.96-7.91\left(1 \mathrm{H}, \mathrm{m}, \mathrm{H}_{\mathrm{Ar}}\right), 7.89\left(1 \mathrm{H}, \mathrm{dd}, J=2.0 \mathrm{~Hz}, J=0.6 \mathrm{~Hz}, \mathrm{H}_{3}\right), 7.83-7.73(2 \mathrm{H}, \mathrm{m}$, $\left.\mathrm{H}_{\mathrm{Ar}}\right), 7.68(1 \mathrm{H}, \mathrm{s}, \mathrm{NH}), 7.61-7.51\left(1 \mathrm{H}, \mathrm{m}, \mathrm{H}_{\mathrm{Ar}}\right), 7.50\left(1 \mathrm{H}, \mathrm{dd}, J=5.3 \mathrm{~Hz}, J=2.0 \mathrm{~Hz}, \mathrm{H}_{5}\right), 4.52$ $\left(2 \mathrm{H}, \mathrm{s}, \mathrm{CH}_{2}\right) ;{ }^{13} \mathrm{C}-\mathrm{NMR}\left(75 \mathrm{MHz}, \mathrm{DMSO}-d_{6}\right) \delta(\mathrm{ppm}) 165.7,150.3,149.7,148.4,138.4,134.0$, 132.6, 131.5, 130.1, 123.0, 118.7, 111.7, 33.3; Anal. Calcd for $\mathrm{C}_{14} \mathrm{H}_{11} \mathrm{~N}_{3} \mathrm{OS}$ (269.32) C, 62.44; H, 4.12; N, 15.60\%. Found: C, 62.35; H, 4.35; N, 15.81\%.

4-(3-Methoxybenzylsulfanyl)pyridine-2-carboxamide (6m). White crystals, yield 30\%, mp. 111-113 ${ }^{\circ} \mathrm{C}$; IR $\left(v_{\max }, \mathrm{cm}^{-1}\right) 3384(\mathrm{~N}-\mathrm{H}), 3293(\mathrm{~N}-\mathrm{H}), 3220(\mathrm{~N}-\mathrm{H}), 1677(\mathrm{C}=\mathrm{O}), 1600\left(\mathrm{NH}_{2}\right)$, $1050\left(\mathrm{OCH}_{3}\right) ;{ }^{1} \mathrm{H}-\mathrm{NMR}\left(300 \mathrm{MHz}, \mathrm{DMSO}-d_{6}\right) \delta(\mathrm{ppm}) 8.41(1 \mathrm{H}, \mathrm{dd}, J=5.3 \mathrm{~Hz}, J=0.6 \mathrm{~Hz}$, $\left.\mathrm{H}_{6}\right), 8.09(1 \mathrm{H}, \mathrm{s}, \mathrm{NH}), 7.90\left(1 \mathrm{H}, \mathrm{dd}, J=2.0 \mathrm{~Hz}, J=0.6 \mathrm{~Hz}, \mathrm{H}_{3}\right), 7.68(1 \mathrm{H}, \mathrm{s}, \mathrm{NH}), 7.48$ (1H, dd, $\left.J=5.3 \mathrm{~Hz}, J=2.0 \mathrm{~Hz}, \mathrm{H}_{5}\right), 7.28-7.22\left(1 \mathrm{H}, \mathrm{m}, \mathrm{H}_{\mathrm{Ar}}\right), 7.04-7.01\left(\mathrm{~m}, 2 \mathrm{H}, \mathrm{H}_{\mathrm{Ar}}\right), 6.85-6.82(1 \mathrm{H}, \mathrm{m}$, $\left.\mathrm{H}_{\mathrm{Ar}}\right), 4.41\left(2 \mathrm{H}, \mathrm{s}, \mathrm{CH}_{2}\right), 3.74\left(\mathrm{~s}, 3 \mathrm{H}, \mathrm{OCH}_{3}\right) ;{ }^{13} \mathrm{C}-\mathrm{NMR}\left(75 \mathrm{MHz}, \mathrm{DMSO}-d_{6}\right) \delta(\mathrm{ppm}) 165.8$, 159.5, 150.5, 150.2, 148.3, 137.8, 129.9, 122.9, 121.2, 118.6, 114.7, 113.1, 55.2, 34.2; Anal. Calcd for $\mathrm{C}_{14} \mathrm{H}_{14} \mathrm{~N}_{2} \mathrm{O}_{2} \mathrm{~S}$ (274.34) C, 61.29; H, 5.14; N, 10.21\%. Found: C, 61.05; H, 5.31; N, $10.45 \%$.

4-(4-Nitrobenzylsulfanyl)pyridine-2-carboxamide (6n). Yellow crystals, yield 60\%, mp. 143$145^{\circ} \mathrm{C}$; IR $\left(v_{\max }, \mathrm{cm}^{-1}\right) 3377(\mathrm{~N}-\mathrm{H}), 3284(\mathrm{~N}-\mathrm{H}), 3185(\mathrm{~N}-\mathrm{H}), 1685(\mathrm{C}=\mathrm{O}), 1601\left(\mathrm{NH}_{2}\right), 1519$ $\left(\mathrm{NO}_{2}\right), 1347\left(\mathrm{NO}_{2}\right) ;{ }^{1} \mathrm{H}-\mathrm{NMR}\left(300 \mathrm{MHz}, \mathrm{DMSO}-d_{6}\right) \delta(\mathrm{ppm}) 8.41(1 \mathrm{H}, \mathrm{dd}, J=5.3 \mathrm{~Hz}, J=0.5$ $\left.\mathrm{Hz}, \mathrm{H}_{6}\right), 8.27-8.13\left(2 \mathrm{H}, \mathrm{m}, \mathrm{H}_{\mathrm{Ar}}\right), 8.08(1 \mathrm{H}, \mathrm{s}, \mathrm{NH}), 7.89\left(1 \mathrm{H}, \mathrm{dd}, J=2.0 \mathrm{~Hz}, J=0.5 \mathrm{~Hz}, \mathrm{H}_{3}\right)$, 7.80-7.68 (2H, m, $\left.\mathrm{H}_{\mathrm{Ar}}\right), 7.68(1 \mathrm{H}, \mathrm{s}, \mathrm{NH}), 7.51\left(1 \mathrm{H}, \mathrm{dd}, J=5.3 \mathrm{~Hz}, J=2.0 \mathrm{~Hz}, \mathrm{H}_{5}\right), 4.62(2 \mathrm{H}, \mathrm{s}$, $\left.\mathrm{CH}_{2}\right) ;{ }^{13} \mathrm{C}-\mathrm{NMR}\left(75 \mathrm{MHz}, \mathrm{DMSO}-d_{6}\right) \delta(\mathrm{ppm}) 165.7,150.4,149.5,148.4,146.9,144.8,130.3$, 123.9, 123.1, 118.8, 33.4; Anal. Calcd for $\mathrm{C}_{13} \mathrm{H}_{11} \mathrm{~N}_{3} \mathrm{O}_{3} \mathrm{~S}$ (289.31) C, 53.97; H, 3.83; N, 14.52\%. Found: C, 53.77; H, 3.95; N, 14.68\%.

4-(3,5-Dinitrobenzylsulfanyl)pyridine-2-carboxamide (6o). Yellowish crystals, yield 35\%, mp.180-182 ${ }^{\circ} \mathrm{C}$; IR $\left(v_{\max }, \mathrm{cm}^{-1}\right) 3388(\mathrm{~N}-\mathrm{H}), 3262(\mathrm{~N}-\mathrm{H}), 3163(\mathrm{~N}-\mathrm{H}), 1676(\mathrm{C}=\mathrm{O}), 1615\left(\mathrm{NH}_{2}\right)$, $1535\left(\mathrm{NO}_{2}\right), 1342\left(\mathrm{NO}_{2}\right)$; ${ }^{1} \mathrm{H}-\mathrm{NMR}\left(300 \mathrm{MHz}, \mathrm{DMSO}-d_{6}\right) \delta(\mathrm{ppm})$ 8.78-8.77 $\left(2 \mathrm{H}, \mathrm{m}, \mathrm{H}_{\mathrm{Ar}}\right), 8.71-$ $8.70\left(1 \mathrm{H}, \mathrm{m}, \mathrm{H}_{\mathrm{Ar}}\right), 8.42\left(1 \mathrm{H}, \mathrm{dd}, J=5.3 \mathrm{~Hz}, J=0.5 \mathrm{~Hz}, \mathrm{H}_{6}\right), 8.07(1 \mathrm{H}, \mathrm{s}, \mathrm{NH}), 7.92(1 \mathrm{H}, \mathrm{dd}, J=$ $\left.2.0 \mathrm{~Hz}, J=0.5 \mathrm{~Hz}, \mathrm{H}_{3}\right), 7.67(1 \mathrm{H}, \mathrm{s}, \mathrm{NH}), 7.56\left(1 \mathrm{H}, \mathrm{dd}, J=5.3 \mathrm{~Hz}, J=2.0 \mathrm{~Hz}, \mathrm{H}_{5}\right), 4.76(2 \mathrm{H}, \mathrm{s}$, $\left.\mathrm{CH}_{2}\right) ;{ }^{13} \mathrm{C}-\mathrm{NMR}$ (75 MHz, DMSO- $d_{6}$ ) $\delta$ (ppm) 165.7, 150.4, 148.9, 148.5, 148.3, 141.8, 129.4, 123.2, 119.1, 117.9, 32.6; Anal. Calcd for $\mathrm{C}_{13} \mathrm{H}_{10} \mathrm{~N}_{4} \mathrm{O}_{5} \mathrm{~S}$ (334.31) C, 46.71; H, 3.02; N, 16.76\%. Found: C, 46.82; H, 3.25; N, 16.59\%.

\section{Antimycobacterial evaluation}

In vitro antimycobacterial activity of the compounds was evaluated against Mycobacterium tuberculosis CNCTC My 331/88, Mycobacterium kansasii CNCTC My 235/80, Mycobacterium kansasii 6509/96, Mycobacterium avium CNCTC My 330/88 and four multidrug-resistant strains of $M$. tuberculosis using the micromethod for the determination of the minimum inhibitory concentration (MIC). M. tuberculosis CNCTC My 331/88, M. kansasii CNCTC My 235/80, and M. avium were obtained from the Czech National Collection of Type Cultures (CNCTC); $M$. kansasii 6509/96 and multidrug-resistant strains were clinical isolates. The activities of the compounds were determined in a Šula semisynthetic medium (SEVAC, Prague). The compounds 
were added to the medium in dimethylsulfoxide solutions. The following concentrations were used: $1000,500,250,125,62,32,16,8,4$ and $2 \mu \mathrm{mol} / \mathrm{L}$. MICs were determined after incubation at $37{ }^{\circ} \mathrm{C}$ for 14 and 21 days, and for M. kansasii for 7, 14, and 21 days. MIC was the lowest concentration of a substance, at which the inhibition of the growth of mycobacteria occurred. INH was used as a standard.

The resistant strains of $M$. tuberculosis may be characterized in this way: M. tuberculosis 735719/98 resistant to isoniazid, rifampicin, streptomycin, ethambutol, ofloxacin, and ansamycin; M. tuberculosis 9449/2007 resistant to isoniazid, rifampicin, streptomycin, and ansamycin; $M$. tuberculosis 234/2005 resistant to isoniazid, rifampicin, streptomycin, ethambutol, and ansamycin; M. tuberculosis 8666/2010 resistant to isoniazid, rifampicin, streptomycin, ethambutol, ofloxacin, ansamycin, and clofazimine.

\section{Antiproliferative and cytotoxic assay}

The target compounds were assayed against cell lines K-562 and HUVEC for their antiproliferative effects and against HeLa for their cytotoxic effects. The cells were incubated with ten concentrations of the test compounds ${ }^{10}$.

Suspension cultures of K-562 in micro plates were analyzed by an electronic cell analyzer system CASY 1 (SCHÄRFE, Reutlingen, Germany) using an aperture of $150 \mu \mathrm{m}$. The software for data evaluation CASYSTAT (SCHÄRFE) offers fast graphical evaluation of the measurement parameters, e.g. as diagrams of cell diameter distributions, overlays of different curves, and cell volume distributions. The $0.2 \mathrm{~mL}$ content of each well in the micro plate was diluted 1:50 with CASYTON (NaCl: $7.93 \mathrm{~g} / \mathrm{L} ; \mathrm{Na}_{2}$ EDTA: $0.38 \mathrm{~g} / \mathrm{L} ; \mathrm{KCl}: 0.4 \mathrm{~g} / \mathrm{L} ; \mathrm{NaH}_{2} \mathrm{PO}_{4}$ monohydrate: $0.22 \mathrm{~g} / \mathrm{L} ; \mathrm{NaH}_{2} \mathrm{PO}_{4}$ dihydrate: $\left.2.45 \mathrm{~g} / \mathrm{L} ; \mathrm{NaF}: 0.3 \mathrm{~g} / \mathrm{L} ; \mathrm{SCHÄRFE}\right)$. Every count $/ \mathrm{mL}$ was automatically calculated from the arithmetic mean of three successive counts of $0.4 \mathrm{~mL}$ each. From the dose response curves the G150 values (concentration which inhibited cell growth by $50 \%$ ) were calculated with CASYSTAT. The G150 value was defined as being where the concentration-response curve intersected the $50 \%$ line, determined by means of the cell counts/mL, compared to control.

The monolayers of the adherent HUVEC and HeLa cells were fixed by glutaraldehyde and stained with a $0.05 \%$ solution of methylene blue for $15 \mathrm{~min}$. After gently washing, the stain was eluted by $0.2 \mathrm{~mL}$ of $0.33 \mathrm{M} \mathrm{HCl}$ in the wells. The optical densities were measured at $630 \mathrm{~nm}$ in a DYNATECH MR 7000 micro plate reader. Comparisons of the different values were performed with Microsoft Excel.

\section{Molecular modeling and QSAR study}

Quantum-chemical calculations were performed using the program Gaussian 03W, version 6.1, revision-E.01 (Gaussian, Inc.). The geometry of the molecules was optimized using the B3LYP/6-311+G(d,p) method. Possible starting conformations for the optimizations at the DFT level were found by the conformational search module of the software HyperChem 8.0.8 (HyperCube, Inc.) on RM1 level. This method involves a random variation of dihedral angles to 
generate new structures and then minimizing the energy of each of these angles. Final models were visualized using the software GaussView, v. 4.1.2; (Gaussian, Inc.).

The octanol-water partition coefficients, expressed as $\log P$ values, were calculated using Advanced Chemistry Development (ACD/Labs) Software V11.02. The $\log P$ values were transformed to the increments $\Delta \log P$ by the subtraction of the value corresponding to the unsubstituted derivative.

Regression analyses were run on a PC computer using the Microsoft Excel program. In the equations, the figures in the parentheses are the standard errors of the regression coefficients, $n$ being the number of compounds, $r$ the correlation coefficient, and $s$ the standard error of estimate. All equations are statistically significant at the $1 \%$ level of probability.

\section{Acknowledgements}

This work was financially supported by project No. MSM 0021620822 of the Ministry of Education of the Czech Republic. We are indebted to Ms I. Vencovská and Assoc. Prof. J. Kuneš (Department of Inorganic and Organic Chemistry, Faculty of Pharmacy, Charles University) for recording IR spectra and NMR spectra, respectively and Mrs. B. Janíčková (Regional Institute of Public Health) for antimycobacterial evaluation.

\section{References}

1. World Health Organization, Stop TB Partnership. Tuberculosis Global Facts 2010. http://www.who.int/tb

2. Gandhi, N. R.; Nunn, P.; Dheda, K. H.; Schaft, S.; Zignol, M.; Van Soolingen, D.; Jensen, P.; Bayona, J. Lancet 2010, 375, 1830.

3. Klimešová, V.; Svoboda, M.; Waisser, K.; Macháček, M.; Buchta, V.; Odlerová, Ž. Arch. Pharm. Pharm. Med. Chem. 1996, 329, 438.

4. Klimešová, V.; Svoboda, M.; Waisser, K.; Pour, M.; Kaustová, J. Collect. Czech. Chem. Commun. 1999, 64, 417.

5. Klimešová, V.; Svoboda, M.; Waisser, K.; Kaustová, J.; Buchta, V.; Králová, K. Eur. J. Med. Chem. 1999, 34, 433.

6. Herzigová, P.; Klimešová, V.; Palát, K.; Kaustová, J.; Dahse, H. M.; Möllmann, U. Arch. Pharm. Chem. Life Sci. 2009, 342, 394.

7. Belda, O.; Moberg, Ch. Synthesis 2002, 11, 1601.

8. Takahiro, I.; Toshiaki, M. Org. Letters 2004, 6, 4587.

9. Exner, O. Correlation Analysis of Chemical Data; Plenum Press: New York, 1988; pp. 6162.

10. Dahse, H. M.; Schlegel, B.; Gräfe, U. Pharmazie 2001, 56, 489. 